\title{
Generalised Model of Multiphase Tesla's Egg of Columbus and Practical Analysis of 3-Phase Design
}

\author{
Atamer Gezer ${ }^{1}$, Mehmet Onur Gülbahçe², Derya Ahmet Kocabaş² \\ 'Ford Otosan R\&D Center, Istanbul, Turkey \\ 2Department of Electrical Engineering, Faculty of Electrical and Electronics Engineering, Istanbul Technical University, Istanbul, Turkey
}

Cite this article as: A. Gezer, M.O. Gülbahçe, D.A. Kocabaş, "Generalised Model of Multiphase Tesla's Egg of Columbus and Practical Analysis of 3-Phase Design", Electrica, vol. 18, no: 2, pp. 151-158, 2018

\begin{abstract}
"Tesla's Egg of Columbus" is the name given to the experiment conducted in 1893 by Nikola Tesla that proved the existence of rotating magnetic field produced by two-phase AC winding. In this study, a generalized mathematical magnetic field model for m-phase "Tesla's Egg of Columbus" is presented in detail, and a novel 3-phase model of "Tesla's Egg of Columbus" is designed analytically by means of generalized expressions. Electrical and geometric quantities are calculated, and the system is analyzed by finite element analysis (FEA). The designed system is manufactured and tested for rated current and for constant and variable frequency with eggs made of different materials. Optimized frequency for materials and geometries are obtained and compared.
\end{abstract}

Keywords: Tesla's Egg of Columbus, 3-phase rotating magnetic field, mathematical model

\section{Corresponding Author:}

Mehmet Onur Gülbahçe

E-mail:

ogulbahce@itu.edu.tr

Received: 13.11.2017

Accepted: 23.03 .2018

(c) Copyright 2018 by Electrica

Available online at

http://electrica.istanbul.edu.tr

DOI: 10.5152/iujeee.2018.1824

\section{Introduction}

Nikola Tesla first introduced rotating field theory to science in 1882. In 1883, he invented the first induction motor that operates by rotating field principle. In 1888 , he secured his patent for his polyphase alternating current motors, generators and rotating filed principles [1]. He named his project "Tesla's Egg of Columbus" which describes the basic principles of rotating magnetic field and induction motors and he presented it to the World Colombian Exhibition in 1893. The name "Egg of Columbus" comes from the real story of Christopher Columbus who asked for ship support from the Queen of Spain for his journey to India. The Queen wanted him to make an egg stand vertical in balance. He broke the sharper edge of the egg, used that side to enable the egg to keep standing vertically, and thus he earned support from the Queen. In Tesla's project, an egg made of copper rotates around a vertical axis while it keeps its balance on a surface placed over the space inside a circular iron core. This proves the existence of rotating magnetic field [1].

The basic operational principle of Tesla's Egg depends on the rotation of total magnetic flux density vectors created at the centre of the stator core which are induced by the magnetomotive force ( $\mathrm{mmf}$ ) created by two phase alternating current winding. With this project, Nikola Tesla managed to keep the copper egg rotating vertically around its main axis with a gyroscopic motion. This provided the effects of rotating field in a practical way. The printed sketch in his paper, published in 1919 in the Electrical Experimenter journal, is shown in Figure 1 [2].

In the project, two separated alternating current windings placed with an angle of $90^{\circ}$ between their axes were wound around a toroidal iron core. Tesla introduced the production of rotating field in the air-gap inside the stator core when two-phase voltage with an electrical phase difference of $90^{\circ}$ was applied to these windings. He placed an oval engraved wooden layer (egg-board) towards the centre over the whole air-gap inside the core. He analysed the 


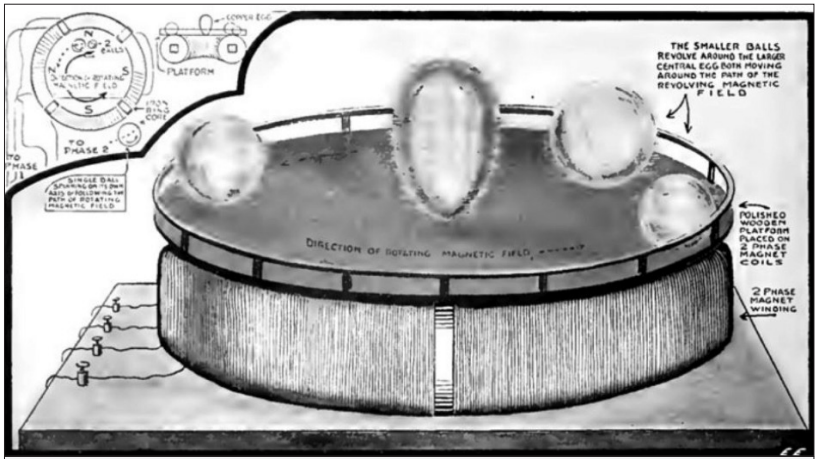

Figure 1. The sketch of Tesla's Egg of Columbus experiment that was published in the journal of Electrical Experimenter in March 1919 [2]

effects of rotating magnetic field on a copper egg, brass balls and iron disks when they were placed on the wood. The supply frequency fluctuated between $25-300 \mathrm{~Hz}$ and the ideal operating frequency was found to be $35-40 \mathrm{~Hz}$ [2].

A design for Tesla's two-phase inductor was given in order to demonstrate a rotating field based on the famous Tesla's Egg of Columbus without establising a generalised model for multiphase structure [3].

In this study, generalised mathematical expression of mmf created inside the air-gap was derived for polyphase windings having been inspired by Tesla's Egg of Columbus that was realised by two-phase ac winding and basic principles of design criteria for $\mathrm{m}$-phases. Starting from this point, a 3-phase version of the system that does not exist in previous studies was designed analytically by calculating the necessary electrical and mechanical quantities. The design was then analysed by Finite Elements Method (FEM). Various rotating objects made of different materials (copper, aluminium, brass) with different geometries (egg, disc) were manufactured. Test setup was installed and was supplied by variable frequency voltage while keeping the current constant. The most efficient geometry, material and supply frequency for a geometry were measured and observed.

\section{Generalised Ampere-Turn Expression of Multi-Phase Tesla's Egg of Columbus}

The idea of installing more than one winding to a single machine paved the way for multi-phase alternating current machines. The proof of rotating field by Tesla's Egg of Columbus opened the way to the design of rotating alternating current machines. In previous studies, only two-phase design details are given without generalising the design criteria [3]. Multi-phase electric motors are more advantagous in terms of power density and utility factor. But as the number of phases increases, the need for multiphase power supplies arises. 3 phase is the accepted common number of phases in the engineering world. Therefore, in this paper, a generalised model is given and practical implementation is performed only for a 3-phase system.
In order to produce a rotating field with $\mathrm{m}$ phase winding, all phase windings have to be placed in a symmetrical geometry and all phase spreads should be of the same widths. Windings must be supplied with a $\mathrm{m}$ phase symmetrical alternating current. In 2 poles $(2 p=2)$ and $m$ phases symmetric structure, a total of $(2 \pi / \mathrm{m})$ radian section for each phase is reserved. For each phase, $(\pi / \mathrm{m})$ radian phase bands are formed by dividing this part into two, which are placed on reverse sides of stator at an angle of $180^{\circ}$. Under each pole, an electrical phase difference between the phase winding axes is created by placing the windings at a geometrical difference of $(2 \pi / \mathrm{m})$ [4-7]. The number of turns of the phases $(\mathrm{N})$ must be equal for symmetry. $\mathrm{N}_{\mathrm{m}}(\theta)$ represents the phase position of $\mathrm{N}$ number of turns for $\mathrm{m}^{\text {th }}$ phase winding (1).

$$
\begin{aligned}
& N_{1}(\theta)=N \\
& N_{2}(\theta)=N \cdot e^{-j \frac{2 \pi}{m}} \\
& N_{3}(\theta)=N \cdot e^{-j \frac{4 \pi}{m}}
\end{aligned}
$$

$\mathrm{M}$

$$
N_{m}(\theta)=N \cdot e^{-\frac{2 \pi(m-1)}{m}}
$$

When the number of poles are 2 , the geometric angle $\left(a_{\text {geo }}\right)$ and electrical angle $\left(a_{\text {elec }}\right)$ are the same. If the number of poles are more than two, then the geometric angle and electrical angle differ from each other and the structure given for two poles is repeated for each pole by accepting the width of each pole "electrically $180^{\circ \prime \prime}$. Geometric angle can be converted into electrical angle by multiplying it with a coefficient of $p$ as in (2) [6]. In general, phase spreads must have a width of $[2 \pi /(\mathrm{m} .2 \mathrm{p})]$ in radians.

$\alpha_{\text {elec }}=\alpha_{\text {geo }} P$

The value and the direction of the ampere-turn (mmf) of one phase winding which is positioned on the axis of phase winding depends on the instantaneous value of the sinusoidal phase current. When $\mathrm{m}$-phase alternating voltage is applied to each phase of the $m$-phase winding with a phase difference of $(2 \pi / \mathrm{m})$ radians, $\mathrm{m}$ different $\mathrm{mmf}$ phasors are located within the gap each having their own instantaneous values [7]. The instantaneous values of the phase currents can be seen in (3). $I$ is the effective value, $I_{m}(\omega t)$ is the the instantaneous value of the $\mathrm{m}^{\text {th }}$ phase.

$$
\begin{aligned}
& I_{1}(\omega t)=\sqrt{2} I \cdot \cos (\omega t) \\
& I_{2}(\omega t)=\sqrt{2} I \cdot \cos \left(\omega t-\frac{2 \pi}{m}\right) \\
& I_{3}(\omega t)=\sqrt{2} I \cdot \cos \left(\omega t-\frac{4 \pi}{m}\right)
\end{aligned}
$$

M

$I_{m}(\omega t)=\sqrt{2} I \cdot \cos \left(\omega t-\frac{2 \pi(m-1)}{m}\right)$ 
Individual and resultant ampere-turn expressions created by one and $m$ phases are given in (4) and (5-6), respectively.

$$
\begin{aligned}
& F_{1}(\theta, t)=N_{1}(\theta) \cdot I_{1}(\omega t) \\
& F(\theta, t)=F_{1}(\theta, t)+F_{2}(\theta, t)+F_{3}(\theta, t)+L \text { L F }_{m}(\theta, t) \\
& F(\theta, t)=N_{1}(\theta) \cdot I_{1}(\omega t)+N_{2}(\theta) \cdot I_{2}(\omega t) \\
& +N_{3}(\theta) \cdot I_{3}(\omega t)+L ~ L ~ N_{m}(\theta) \cdot I_{m}(\omega t)
\end{aligned}
$$

When (1) and (2) are substituted in (5), (7) is obtained for the resultant $\mathrm{mmf}$ by means of necessary trigonometric (Euler) transformations [4].

$$
F(\theta, t)=\frac{m}{2} N I . e^{j \omega t}
$$

As can be seen in (7), the amplitude of total ampere-turn does not change according to time while the position of resultant magnetic field changes. The complex position vector represents the change of position. This vector accepts the centre of stator as the origin of the coordinate system. The geometrical locus of the end-point of the field vector is a circle. The expression in (7) is the mathematical expression of the rotating magnetic field that is produced by $\mathrm{m}$-phase winding.

The speed of the rotating field is called synchronous speed $\left(n_{s}\right)$. As the currents of $\mathrm{m}$-phase winding complete one period, the rotating field travels along two poles. When there are more than 2 poles, the rotating field completes its path by $\mathrm{f} / \mathrm{p}$ times in seconds. Since the general unit for rotation is rpm or $\mathrm{min}^{-1}$, synchronous speed can be calculated in terms of (8).

$$
n_{s}=\frac{60 \cdot f}{p}
$$

When a closed loop made of conductive material (coils, cast iron, disk, etc.) is placed inside a rotating magnetic field that is generated by the currents flowing through the $\mathrm{m}$-phase stator windings, voltage is induced in the conductive object due to the relative motion. Current flows through the conductive material via closed paths and force is induced on the current carrying conductive material. A tangential force is applied to the conductors to force the material to rotate by the rotating field. Vectorel interattraction between magnetic field provided by the induced currents and magnetic field provided by stator currents causes a torque production on the rotating object. When using conductive materials such as an egg, spin top, disk or a coil wound around a cylinder, rotation begins. The egg, spin top, disk type materials can stand upright by the centrifugal force induced.

\section{Design of the Three Phase Tesla's Egg of Columbus}

Basically, generalized electric machine design consists of a twostep recursive method. The first step includes the obtainment of main dimensions, winding arrangement, etc. by assumptions, and the second step includes the necessary corrections and experimental verification studies [3]. Electromagnetic design will start with determination of the magnetic flux and current density under the fixed magnetic core dimensions and required number of turns will be calculated. The procedure is completed after validation of the proposed air-gap flux density by finite element analysis (FEA).

In this paper, a three-phase and two-pole version of Tesla's Egg of Columbus is designed and design steps are given. Phase spreads for one phase is $60^{\circ}$ as explained above.

\section{Design of Magnetic Circuit and Calculation of Air-Gap Reluctance}

The laminated magnetic core to be used is toroidal shaped with its geometric dimensions given in Table 1 and the top and side views shown in Figure 2 where " $D$ " represents the outer diameter of the core, " $d$ ", the inner diameter, " $h$ "and "a", the cross-section height and width, respectively.

Stator top view of three phase Tesla's Egg of Columbus, including one phase winding that is wound around the core along its phase spread, and winding arrangement for two poles are given in Figure 3. In order to accommodate three phase windings, the stator is divided into 6 phase bands of $60^{\circ}$. Each coil is wound in reverse directions but connected in series and two windings are complementary to each other. These two windings produce equal and opposite magnetic fields with respect to each other.

The magnetic circuit has symmetry and the total magnetic flux within the air-gap $\left(\Phi_{g}\right)$ is the sum of the equal fluxes $\left(\Phi_{\mu}\right)$ produced by winding parts on opposite sides of the core. By assuming the permeability of magnetic core as infinite, the equivalent magnetic circuit in Figure 4 is obtained.

By assuming the flux to be equal within the air-gap, the circuit becomes easier to solve and the air-gap reluctance $\left(\Re_{g}\right)$ and the air-gap flux $\left(\Phi_{\mathrm{g}}\right)$ must be calculated using the magnetic circuit dimensions. Since the air-gap flux produced by one phase winding passes through the air-gap with inconstant width, the total reluctance of the gap must be calculated by evaluating integral. The mean value of the cross-section area is used for ap-

Table 1. Core Dimensions

\begin{tabular}{llc}
\hline Symbol & Unit & Value \\
\hline Outer diameter of the core (D) & $\mathrm{mm}$ & 300 \\
\hline Inner diameter of the core (d) & $\mathrm{mm}$ & 170 \\
\hline cross-section height (h) & $\mathrm{mm}$ & 80 \\
\hline cross-section width (a) & $\mathrm{mm}$ & 65 \\
\hline
\end{tabular}




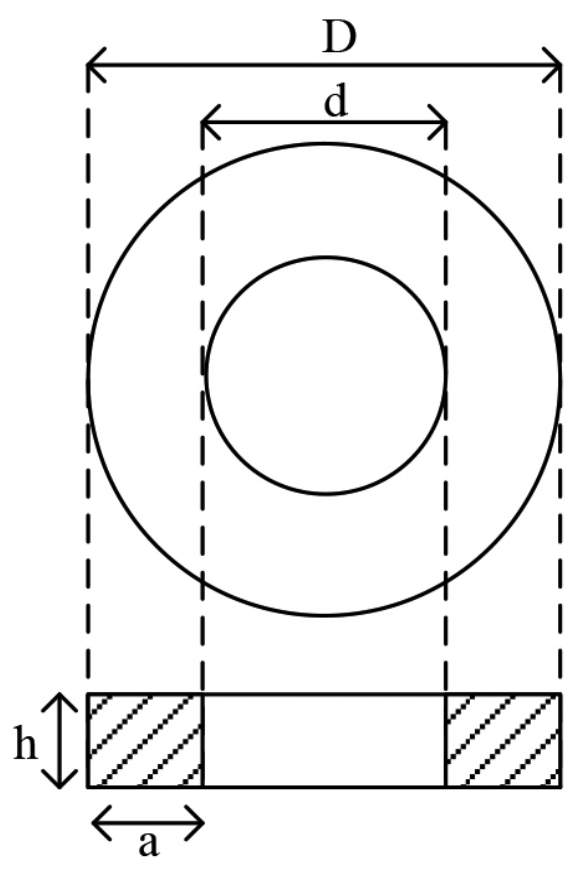

Figure 2. Top and side view of magnetic core

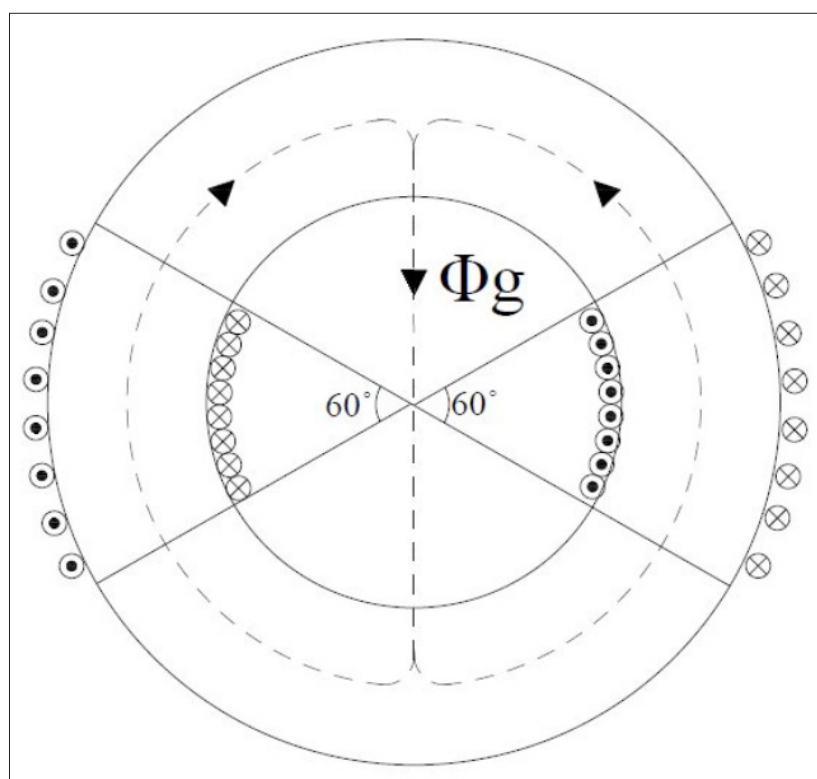

Figure 3. Magnetic Circuit and winding positions for one phase

proximate calculation. The air-gap through the toroid is sliced into $\mathrm{k}$ pieces by $1 \mathrm{~cm}$ and the cross-section area of each slice is calculated by multiplying the toroid height by the width of $\mathrm{i}^{\text {th }}$
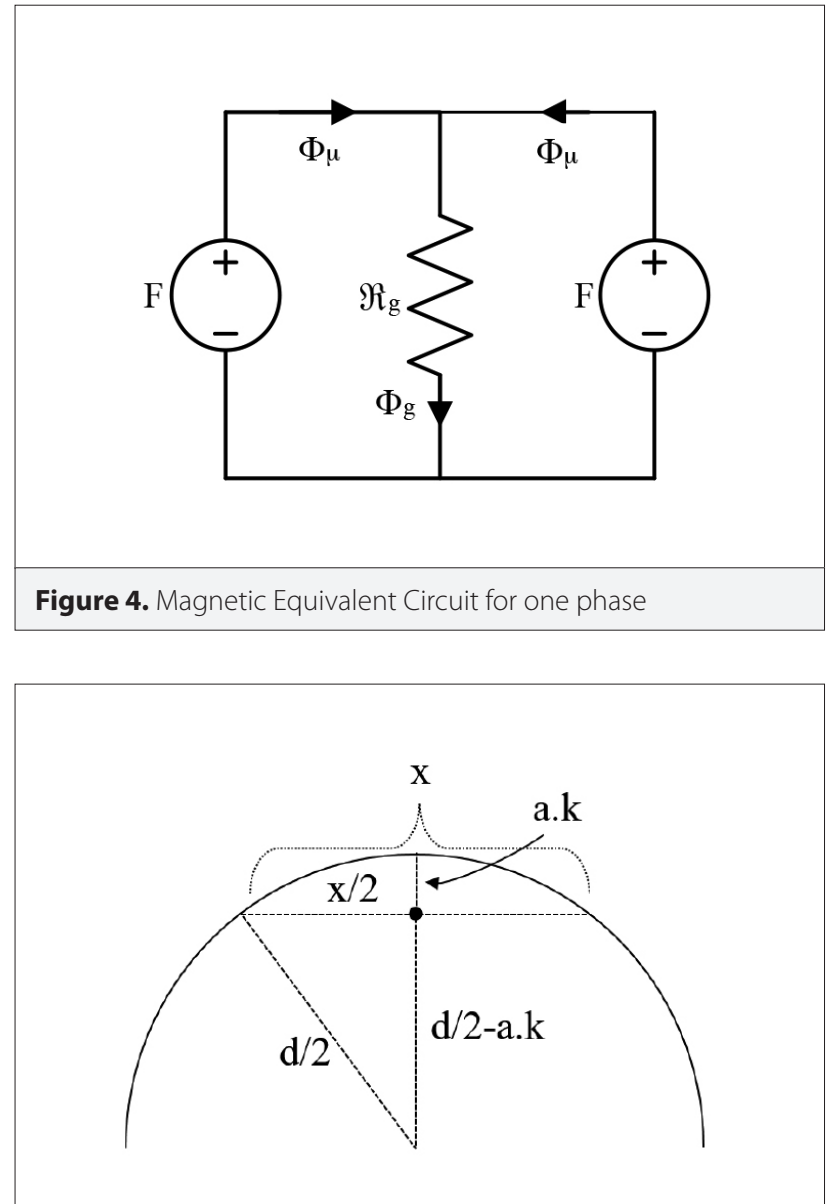

Figure 5. Calculation of width of a. slice when diameter is divided into k parts in order to obtain average air-gap crossection area $(a=1,2, \ldots k)$

slice $(i=1,2, \ldots k)$ given in (9). The sum of the slice sections and the arithmetic average of all sections are calculated as in (10). The drawing used for calculating the mean value of air-gap area is given in Figure 5.

The width of $a^{\text {th }}$ slice in $\mathrm{k}$ slices is calculated by means of trigonometric relations in (9).

$$
x_{a}=2 \sqrt{d \cdot a \cdot k-a^{2} \cdot k^{2}}
$$

According to (9), the average cross-sectional area of the air-gap $\left(A_{\text {g-mean }}\right)$ is calculated by (10).

$$
A_{\text {gort }}=\frac{h \cdot \sum_{a=1}^{k-1} x_{a}}{k-1}
$$

The circuit shown in Figure 4 can be solved by calculating the total magnetic resistance related to the reluctance equation given in (11), and the average value of the air-gap magnetic flux density can be calculated as in (12). 


$$
\begin{aligned}
& \Re_{g}=\frac{F}{\Phi_{g}} \\
& B_{g}=\frac{\Phi_{g}}{A_{g o r t}}
\end{aligned}
$$

\section{Validation of Air-Gap Flux and Calculation of Number of Turns Per Phase}

Before calculating the number of turns per phase, the values of current density and air-gap magnetic flux density must be chosen. The current density was accepted as $4 \mathrm{~A} / \mathrm{mm}^{2}$ and the magnetic flux density $\left(B_{g}\right)$ in the air-gap as $400 \mathrm{mT}$. The conductor dimensions and rated current are determined according to the selected current density. The number of turns per phase was calculated as 250 by using (7) and (11) for the selected initial conditions. The location of the calculated number of turns on the magnetic core is given in Figure 6. For the other two phase windings, conductor distribution is shifted $120^{\circ}$ geometrically which is already equal to electrical angle since the design has two poles.

\section{Finite Element Analysis of the 3 phase-Tesla's Egg of Columbus}

In this section, the design that is composed of the core and windings is imported to a finite element analysis (FEA) software after being sketched in a 3D drawing tool. This design is analysed by FEM while the windings are supplied by a current source at $50 \mathrm{~Hz}$. During the numerical magnetic analysis, the egg and the egg-board is neglected depending on their magnetic properties. The used structure for the analysis is given in Figure 7 with the neglected egg and the egg-board in order to help in understanding.

After the analysis, the existence of the rotating field and the value of the calculated air-gap field is confirmed. As a visualised result, an instantaneous distribution and vectorial representation of magnetic flux density at the instant of $\omega t=3 \pi / 4$ is given in Figure 8, 9; respectively.

After the analysis result confirmed the calculated flux density within the air-gap, the manufacturing stage was started.

\section{Manufacturing of The Three Phase Tesla's Egg of Columbus}

The manufacturing stage of The Three Phase Tesla's Egg of Columbus has four parts, namely productions of the toroidal core, egg and egg-board and winding the coils around the core [8].

The core is produced using laminated steel. The windings are wound around the core in phase spreads of three phases using a toroidal winding machine. The air-gap is covered by the placed egg-board made of pertinax. The egg-board is an oval engraved wooden layer (egg-board) situated towards the cen-

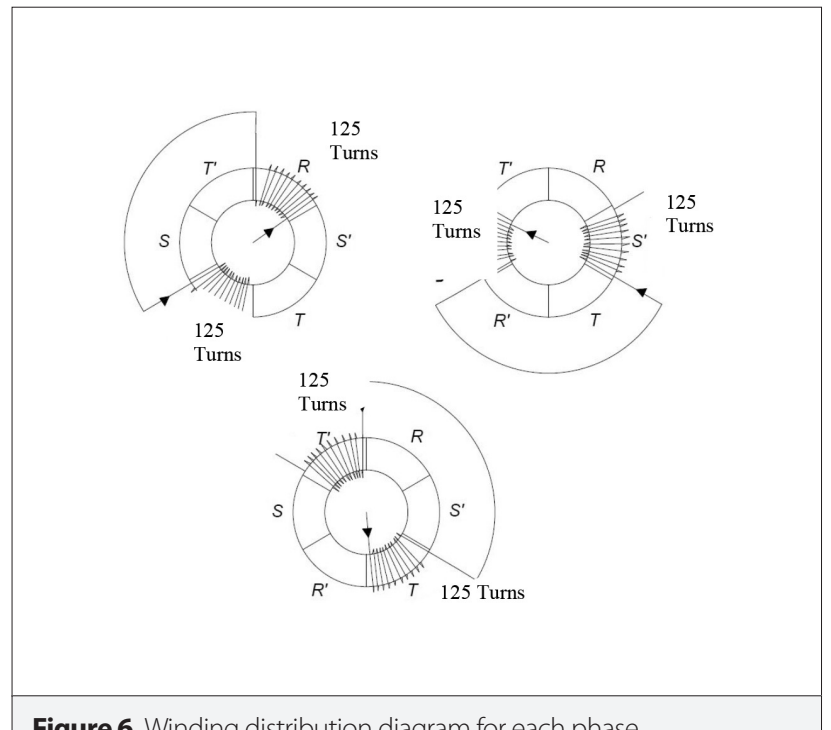

Figure 6. Winding distribution diagram for each phase

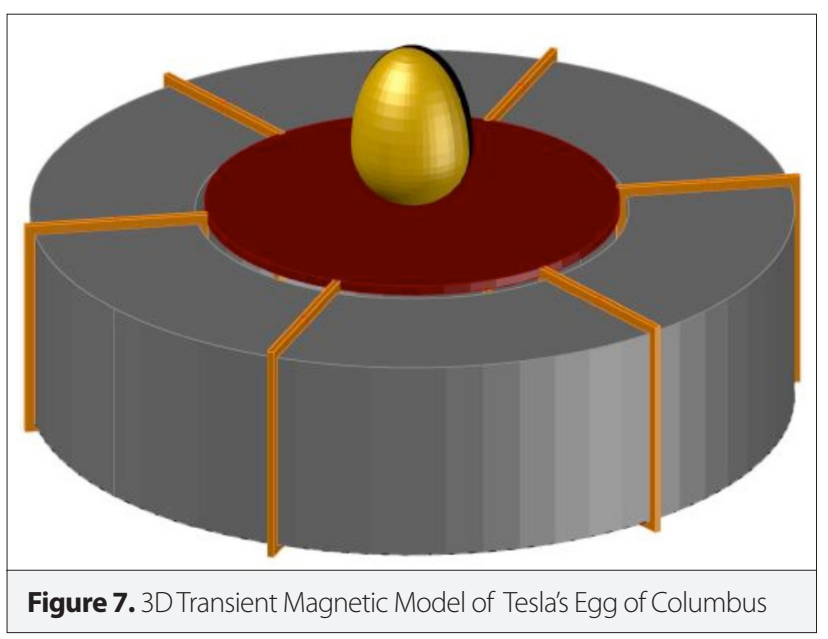

tre of the whole air-gap inside the core. This engraving helps the egg to be exposed to a more magnetic field. It also improves the stability of the egg during rotation.

The dimensions of the egg are inspired from a previous design for one phase and are given in Figure 10 [8]. For an easier production, the egg is manufactured in two pieces that are the top and the bottom parts. This kind of production also helps to increase the weight of the egg by fitting additional pieces made of the same material.

The final product for the Three Phase Tesla's Egg of Columbus is given in Figure 11.

\section{Laboratory Stage}

The test stage is performed in two sub-stages, namely constant and variable frequency supply at the calculated rated current of the system. 


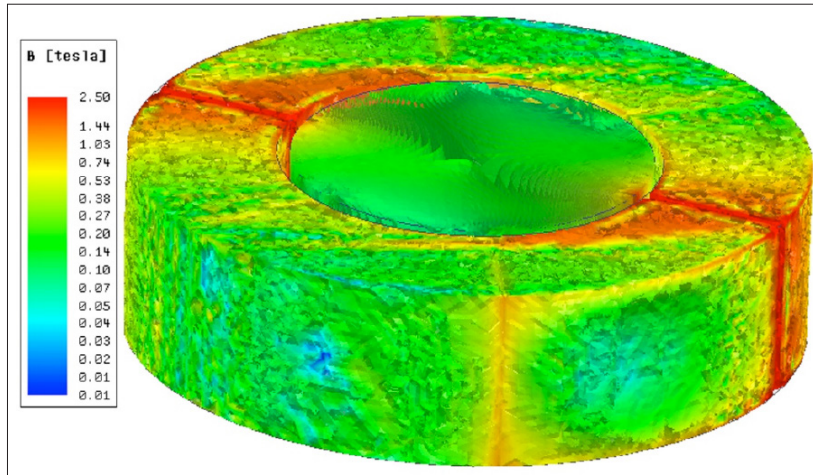

Figure 8. Instantaneous Distribution of magnetic flux density at $\omega t=3 \pi / 4$

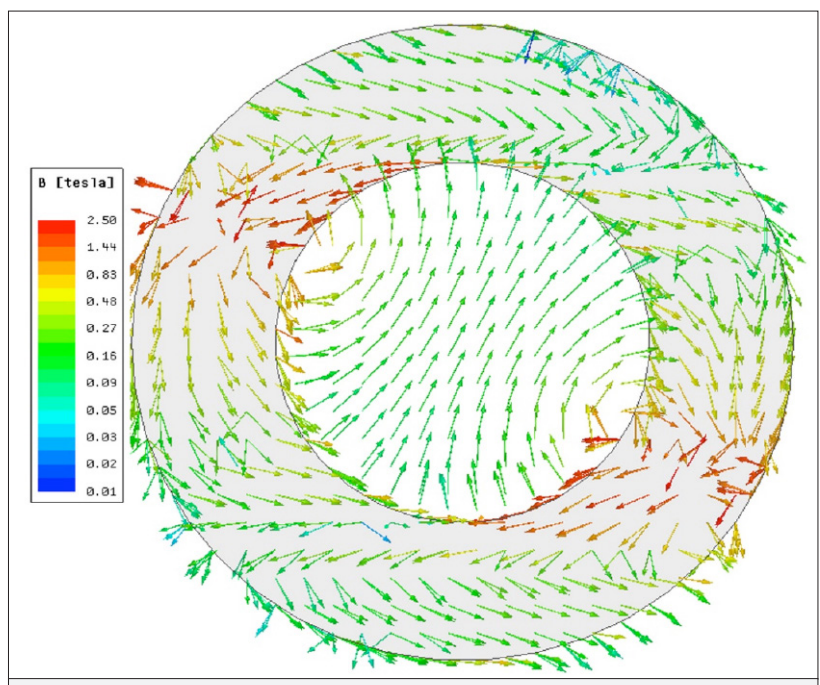

Figure 9. Distribution of magnetic flux density vectors at $\omega t=3 \pi / 4$

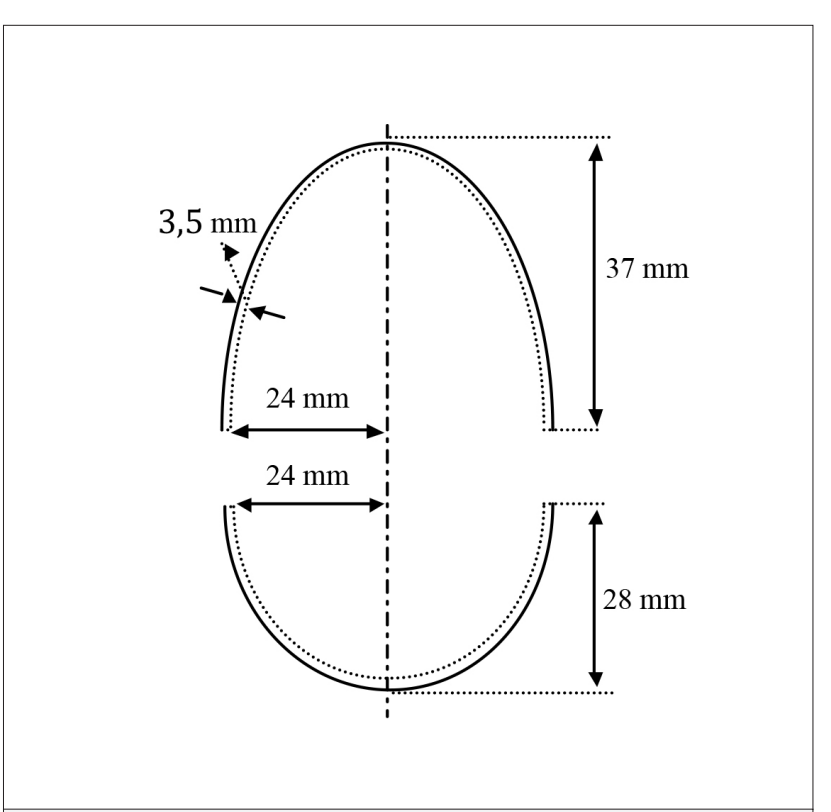

Figure 10. 2D sketch of the Egg

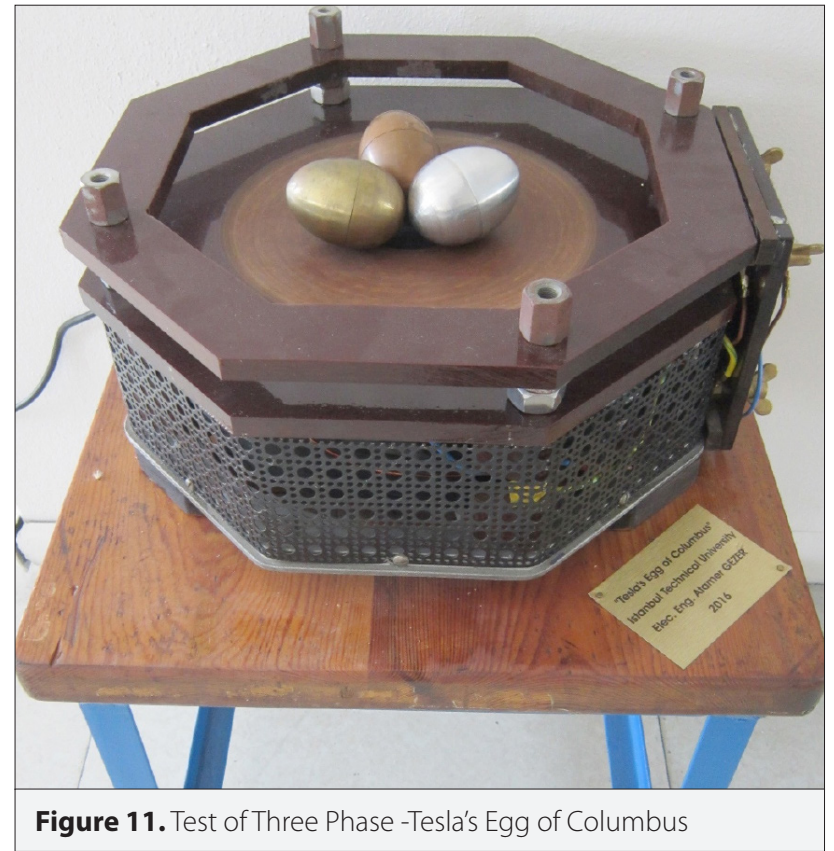

\section{Test for Constant Supply Frequency}

In this test, windings are supplied by $5 \mathrm{~A}$ of rated current at 50 $\mathrm{Hz}$ supply frequency via an

autotransformer. The set used for the constant supply frequencies is given in Figure 12. For low levels of current, insufficient rotational torque is induced resulting in a rotation at low levels of angular speed on horizontal axis. For current levels close to rated current, a rotational torque at higher levels of angular speed that is high enough to keep the egg rotating in the vertical axis is induced.

Different rotational performances were recorded for aluminium disc, aluminium egg and copper egg.

\section{Test of Variable Supply Frequency}

In this test, rated current is applied to windings by a synchronous generator, and the rotational performances of the conducting objects are compared for variable frequency and voltage values between $30 \mathrm{~Hz}$ and $70 \mathrm{~Hz}$. The set used for the variable supply frequencies is given in Figure 13.

The aim of this test is to determine the effect of frequency on the objects to obtain the most ideal rotational motion "without visual wobbling". Visually, it was observed that the ideal value of the frequency of copper and aluminium eggs is $60 \mathrm{~Hz}$. Also no significant change for the aluminium disk for different supply frequencies was recorded. The reason for this is the greater torque generated over the large diameter of the disc by linking more magnetic flux. 

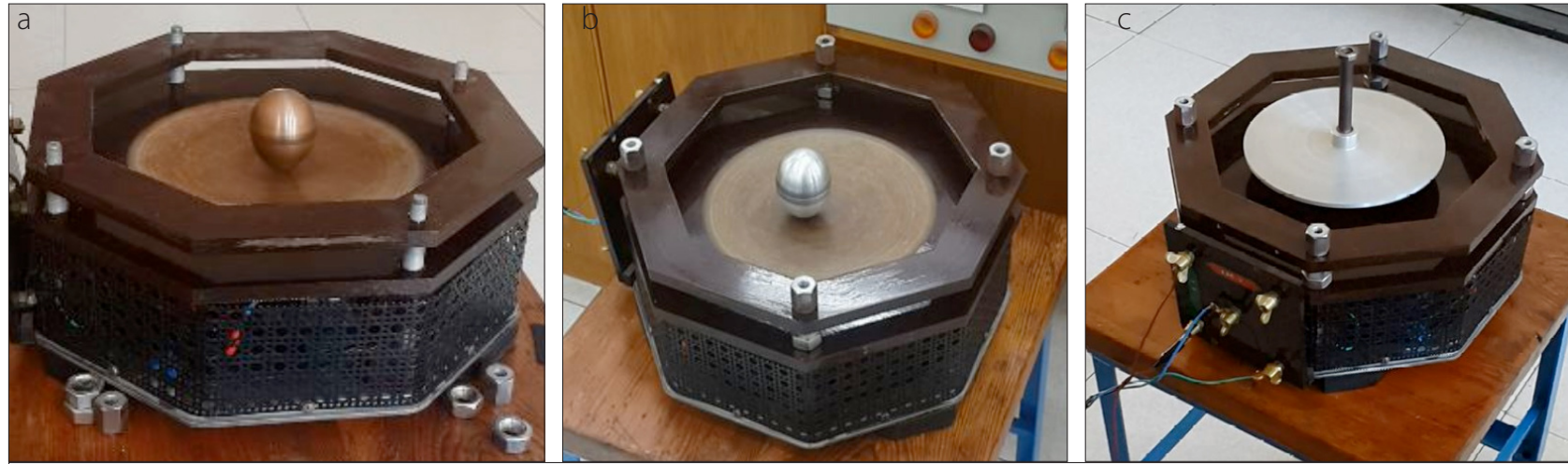

Figure 12. a-c. Rotational behaviour for different objects made of different materials in different geometrical shapes Copper egg (a); Aluminum egg (b); Aluminum disc (c)

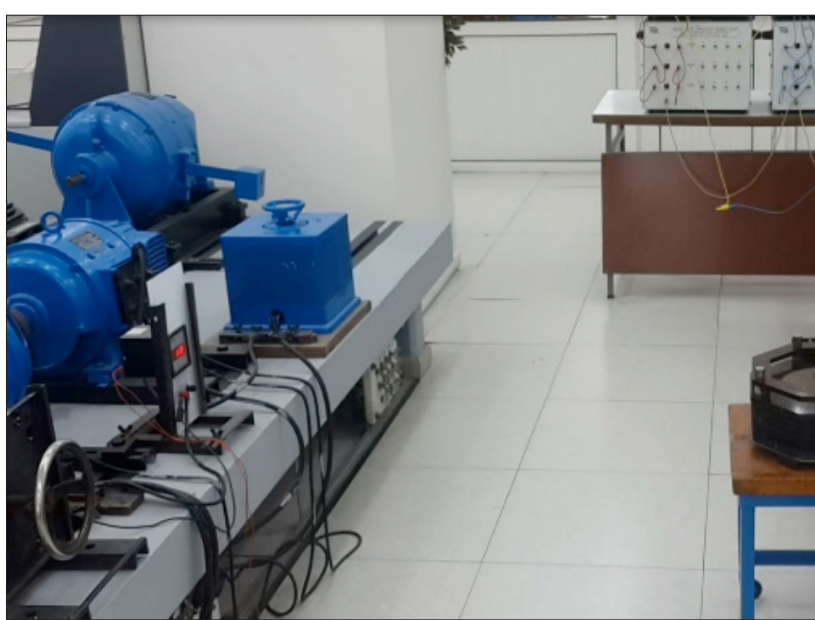

Figure 13. The set used for the tests for Supplying by Variable Frequency and Voltage

\section{Conclusion}

In this study, a generalised mathematical model was created for the project called "Egg of Columbus" which, when the theory was introduced by Tesla, was intended only for two phase. A three-phase version, which does not exist in the literature, was designed analytically. Calculated design information was confirmed by numerical simulations based on FEM.

In the manufacturing stage, designed windings were wound around a laminated toroid and a pertinaks board was placed over the air-gap inside the toroid to enable the egg to rotate firmly. Copper and aluminium eggs and an aluminium disc were manufactured to use as rotating objects. Tests were performed to obtain the effect of the geometry and the material while the coils were supplied by the calculated rated current at constant and variable frequencieAmong all the materials, the most efficient rotation was obtained for aluminium. The best rotational performance was obtained for $60 \mathrm{~Hz}$ for the egg made of aluminium. Aluminium disc was seen to be the most stable and firmly rotating geometry among the objects due to higher linked magnetic flux.

Peer-review: Externally peer-reviewed.

Conflict of Interest: The authors have no conflicts of interest to declare.

Financial Disclosure: The authors declared that this study has received no financial support

\section{References}

1. N. Tesla, "Electro-Magnetic Motor", US Patent Office, Aachen, US381968, 1888.

2. N. Tesla, "Tesla's Egg of Columbus". MECHATRONIKA, Electrical Experimenter, 6, 774-808, 1919.

3. M.L. Dorde, R.M. Petar, "Design of Tesla's Two-Phase Inductor ", International Symposium on Industrial Electronics, Banja Luka- Bosnia and Herzegovina, 115-122, 2014.

4. A.E. Fitzgerald, C.J. Kingsley. "Electric Machinery", The McGraw-Hill, USA, 2003.

5. S.J. Chapman, "Electric Machinery Fundamentals", The McGraw-Hill, USA, 2011.

6. A.F. Mergen, D.A. Kocabas "Windings in Electrical Machines", Birsen Publisher, Istanbul, Turkey 2012.

7. A.F. Mergen, S. Zorlu "Electrical Machines-II", Birsen Publisher, Istanbul, Turkey 2009.

8. "Tesla's Egg of Columbus-Production of working replicas". http:// www.rt17.hr/teslas-egg-of-columbus/. (16.04.2016). 
Atamer Gezer received the B.S. degree in electrical engineering from Istanbul Technical University, Istanbul, Turkey, in 2016. He is a student the M.Sc. degree from 2018 in Electrical Engineering Programme, Institute of Science and Technology, ITU. He is also an Electrical Distribution System Engineer on heavy commercial vehicles harness design, Ford Otosan since 2016. His main subjects of concern are electrical machines, low voltage power distribution system and harness components about automotive.

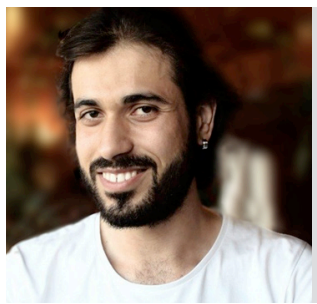

Mehmet Onur Gulbahce received the B.S. degree in electrical engineering from Istanbul University, Istanbul, Turkey, in 2010. He received the M.Sc. degree in 2013 in Electrical Engineering Programme, Institute of Science and Technology, ITU and since 2013 he has been a Ph.D. student in the same programme. He is also a research assistant in the Department of Electrical Engineering, Electrics and Electronics Faculty, ITU since 2011. His main subjects of concern are high-speed electrical machines, drive system and dc-dc converters.

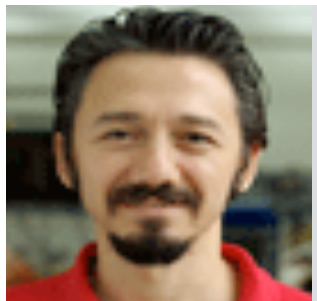

Derya Ahmet Kocabas received the B.S. degree in electrical engineering from ITU, Istanbul, Turkey, in 1994. He received the M.Sc. and Ph.D. degrees from Electrical Engineering Programme, Institute of Science and Technology, ITU, in 1997 and 2004, respectively. His main subjects of concern are design and control of electrical machines, space harmonics, drive systems and power electronics. He joined to Department of Electrical Engineering, Electrics and Electronics Faculty, ITU in 1995 and since January 2009 he has been an Assistant Professor. 